\title{
Vortex Phase Diagram for Mesoscopic Superconducting Disks
}

\author{
V. A. Schweigert,* F. M. Peeters, ${ }^{\dagger}$ and P. Singha Deo \\ Departement Natuurkunde, Universiteit Antwerpen (UIA), B-2610 Antwerpen, Belgium
}

(Received 7 April 1998)

\begin{abstract}
Solving numerically the 3D nonlinear Ginzburg-Landau (GL) equations, we study equilibrium and nonequilibrium phase transitions between different superconducting states of mesoscopic disks which are thinner than the coherence length and the penetration depth. We have found a smooth transition from a multivortex superconducting state to a giant vortex state by increasing both the disk thickness and the magnetic field. A vortex phase diagram is obtained which shows, as a function of the magnetic field, a reentrant behavior between the multivortex and the giant vortex state. [S0031-9007(98)07232-9]
\end{abstract}

PACS numbers: 74.25.Ha, 73.20.Dx, 74.60.Ec

Recently, mesoscopic superconductivity has attracted much attention in view of phase transitions in confined systems with sizes comparable to the coherence $(\xi)$ and penetration $(\lambda)$ lengths. While the type of bulk superconductors is determined only by the value of the Ginzburg-Landau parameter $\kappa=\lambda / \xi$, the experimental observations [1] and the numerical simulations [2,3] of magnetization of mesoscopic thin disks have shown that the type and the order of those transitions between different superconducting states and between the superconducting and the normal state depends crucially on the disk radius $R$ and the thickness $d$. By increasing the disk radius the second-order reversible phase transition observed for small disk radii is replaced by first-order transitions with jumps in the magnetization. In previous theoretical investigations [2,3] only the giant vortex states with fixed total angular momentum $L$ were considered. It is well known [4] that for type-II superconductors $(\kappa>1 / \sqrt{2})$, the triangular Abrikosov vortex lattice is energetically favorable in the range $H_{c 1}<H<$ $H_{c 2}$. Since the effective London penetration depth $\Lambda=$ $\lambda^{2} / d$ increases considerably in thin disks and for $d \ll \lambda$ one would expect the appearance of the Abrikosov multivortex state even in disks made from a material with $\kappa<$ $1 / \sqrt{2}$, such as, e.g., the $A l$ disks studied in Refs. [2,3]. By analogy with classical particles confined by an external potential [5], the structure of a finite number of vortices should differ from a simple triangular arrangement and allow for different metastable states. Using the London approximation Fetter [6] calculated the critical field $H_{c 1}$ for flux penetration into a disk. For a superconducting cylinder the multivortex clusters, containing up to four vortices, were simulated by Bobel [7]. Using the method of images and the London approximation, Buzdin and Brison [8] have considered vortex structures in small $R \ll \Lambda$ disks and found a classical particle ringlike arrangement [5] of vortices. The reverse situation of a lattice of microholes was studied experimentally in Ref. [9] and the configuration of vortices was found. In the present Letter we study the transition from the giant vortex state to this multivortex configuration for thin superconducting disks within the nonlinear Ginzburg-Landau (GL) theory.
We consider a superconducting disk immersed in an insulator media with a perpendicular uniform magnetic field $H_{0}$. For thin disks $(d \ll \xi, \lambda)$ we found [2,3] that it is allowed to average the GL equations over the disk thickness. Using dimensionless variables and the London gauge $\operatorname{div} \vec{A}=0$ for the vector potential $\vec{A}$, we write the system of GL equations in the following form:

$$
\begin{gathered}
\left(-i \vec{\nabla}_{2 \mathrm{D}}-\vec{A}\right)^{2} \Psi=\Psi\left(1-|\Psi|^{2}\right), \\
-\triangle_{3 \mathrm{D}} \vec{A}=\frac{d}{\kappa^{2}} \delta(z) \vec{j}_{2 \mathrm{D}}, \\
\vec{j}_{2 \mathrm{D}}=\frac{1}{2 i}\left(\Psi^{*} \vec{\nabla}_{2 \mathrm{D}} \Psi-\Psi \vec{\nabla}_{2 \mathrm{D}} \Psi^{*}\right)-|\Psi|^{2} \vec{A},
\end{gathered}
$$

with the boundary condition $\left.\left(-i \vec{\nabla}_{2 \mathrm{D}}-\vec{A}\right) \Psi\right|_{r=R}=0$. Here the distance is measured in units of the coherence length $\xi$, the vector potential in $c \hbar / 2 e \xi$, and the magnetic field in $H_{c 2}=c \hbar / 2 e \xi^{2}=\kappa \sqrt{2} H_{c}$. The disk is placed in the plane $(x, y)$, the external magnetic field is directed along the $z$ axis, the indices 2D and 3D refer to two- and three-dimensional operators, and $\vec{j}_{2 \mathrm{D}}$ is the density of the superconducting current. To solve the system of Eqs. (1) and (2) we apply a finite-difference representation of the order parameter and the vector potential on a uniform Cartesian space grid $(x, y)$, with a typical grid spacing of $0.15 \xi$, and use the link variable approach [10]. To find the steady-state solution of the GL equations we add to the left-hand side of Eqs. (1) and (2) the time derivatives of the order parameter and the vector potential, respectively, and use an iteration procedure based on the Gauss-Seidel technique to find $\Psi$. The vector potential is obtained with the fast Fourier transform technique where we set $\vec{A}_{|x|=R_{s},|y|=R_{s}}=H_{0}(x,-y) / 2$ at the boundary of a larger space grid $\left(R_{s}=4 R\right)$.

The giant vortex state is characterized by the total angular momentum $L$ through $\Psi=\psi(\rho) \exp (i L \phi)$, where $\rho$, $\phi$ are the cylindrical coordinates. An arbitrary superconducting state is generally a mixture of different angular harmonics. Nevertheless, we can introduce an analog to the total angular momentum which is still a good quantum number. Choosing circular loops at the periphery 
of the disk we find that the effective angular momentum $L=\Delta \phi / 2 \pi$ does not depend on the loop radii $\rho_{l}$ when it is in some range $\rho_{l}=(0.8-1) R$. This allows us to characterize unambiguously the different superconducting states, where the effective angular momentum is in fact nothing else then the number of vortices in the disk.

To find the different vortex configurations, which include the metastable states, we search for the steadystate solutions of Eqs. (1) and (2) starting from different randomly generated initial conditions. Then we slowly increase and/or decrease the magnetic field and recalculate each time the exact vortex structure. We do this for each vortex configuration in a magnetic field range where the number of vortices stays the same. By comparing the dimensionless Gibbs free energies (of the different vortex configurations) $F=V^{-1} \int\left[2\left(\vec{A}-\vec{A}_{0}\right) \vec{j}_{2 \mathrm{D}}-\right.$ $\left.|\Psi|^{4}\right] d \vec{r}$, where integration is performed over the disk volume $V$, and $\vec{A}_{0}$ is the vector potential of the external uniform magnetic field, we found the ground state. If the system has sufficient time to equilibrate, the system will be in the ground state and we obtain phase transitions between different ground states. If the latter condition is not satisfied the system can remain in a metastable state until this state disappears or becomes unstable with respect to small perturbations in the order parameter and/or the magnetic field. Such nonequilibrium phase transitions lead to hysteresis in the magnetization. The barriers separating the metastable and the ground state are discussed in Refs. [11,12]. Instead of finding the barrier heights, which is a more cumbersome problem, we search for the critical magnetic fields corresponding to the disappearance of such barriers. Here, we started from the Meissner (normal) state and slowly increased (decreased) the magnetic field (typically with steps of $0.01 H_{c 2}$ ) from the zero (nucleation) magnetic field.

The free energies of the different vortex configurations are shown in Fig. 1 for zero disk thickness and for two disk radii: (a) $R=4 \xi$ and (b) $R=4.8 \xi$. For $R=4 \xi$, the vortex configuration can consist of up to six vortices which are arranged on the edge of an ideal polygon. The pentagon and hexagon vortex clusters are always metastable states for $R=4 \xi$. With increasing disk radius the allowed number of vortices increases leading to the appearance of polygons with a vortex inside it when $L>7$. But these structures have a larger energy as compared to the ideal polygons. This result differs from the London approximation of Ref. [8] where the closed packed structures are more preferable even for $L \geq 6$, which clearly shows the limited validity of the approach of Ref. [8]. Another unexpected feature is observed when the magnetic field is further increased. While the model of classical particles [8] predicts a decrease in the intervortex distance followed by the appearance of a new vortex, our simulations show, as a rule, a gradual transition from a multivortex state to a giant vortex state (Fig. 2). In principle, the latter may be a metastable state. The positions of these transitions are indicated

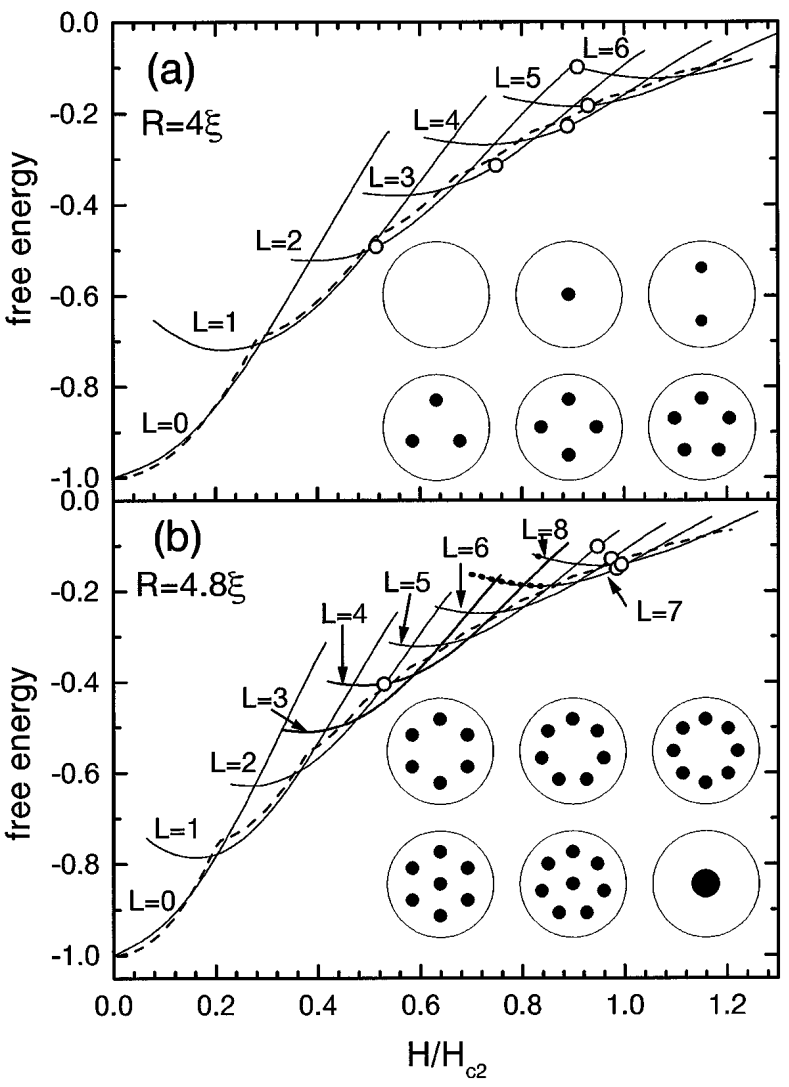

FIG. 1. The free energy of configurations with different number of vortices $L$ for two disk radii $R=4 \xi$ (a) and $R=4.8 \xi$ (b) for zero disk thickness and $\kappa=0.28$. The open circles indicate the transition from a multivortex to a giant vortex state. The latter is located on the right side of the circle. The dotted curves correspond to the free energy of metastable configurations with a vortex inside a polygon for $L=7,8$ (b). The dashed curves are the results of our approximate analytical calculations for the ground state. The insets show the possible multivortex configurations.

by circles in Fig. 1. For a larger disk radius $R=4.8 \xi$, we also observe transitions between different multivortex states. After performing a Fourier analysis of the order parameter we find that a multivortex state corresponding to an ideal polygon presents a mixture of harmonics $\Psi \approx \psi_{0}(\rho)+\sum_{k} \psi_{k}(\rho) \exp (i k L \phi)$ with a rather small contribution of higher $(k>2)$ harmonics. This allows us to find approximately the free energy of different vortex configurations in thin disks $R \ll \Lambda$ where we can neglect the distortion of the magnetic field. For this purpose we take the order parameter as a superposition of only two states $\Psi \approx C_{0}^{1 / 2} \zeta_{0}(\rho)+C_{L}^{1 / 2} \zeta_{L}(\rho) \exp (i L \phi)$, where $\zeta_{0}, \zeta_{L}$ are the eigenfunctions of the linearized first GL equation (1) for different angular momenta $[3,13]$. Substituting this expansion into Eq. (1) we obtain the following set of nonlinear equations for the coefficients $C_{0}$ and $C_{L}$ :

$$
\begin{aligned}
& \lambda_{0} C_{0}=a_{11} C_{0}^{2}+a_{12} C_{0} C_{L}, \\
& \lambda_{L} C_{L}=a_{12} C_{0} C_{L}+a_{22} C_{L}^{2},
\end{aligned}
$$




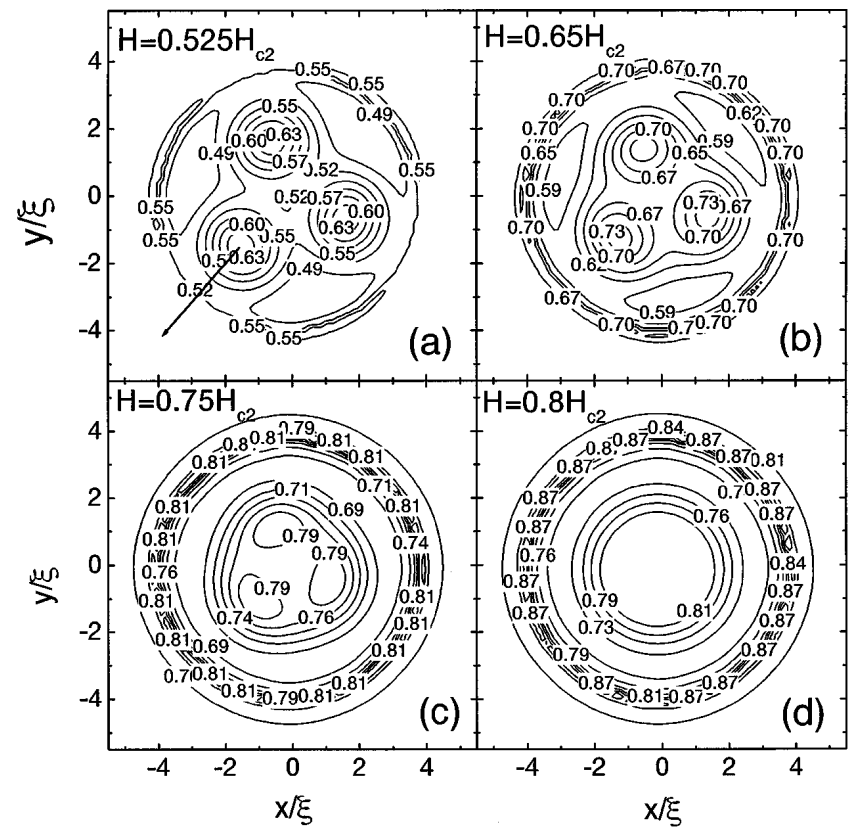

FIG. 2. Contour plot of the magnetic field distribution in the disk plane $(z=0)$ for the case of a three vortex state and for different applied magnetic fields $H_{0}=0.525 H_{c 2}$ (a), $H_{0}=0.65 H_{c 2}$ (b), $H_{0}=0.75 H_{c 2}$ (c), and $H_{0}=0.8 H_{c 2}$ (d). We took $R=4 \xi$ and $d=0.5 \xi \kappa^{2}$. For the lowest magnetic field (a) the three vortex configuration is metastable and a small decrease in the magnetic field leads to a transition to the two vortex state due to the expulsion of a vortex through the disk boundary indicated by the arrow in (a).

with $a_{11}=\left\langle\zeta_{0}^{2} \mid \zeta_{0}^{2}\right\rangle, a_{12}=2\left\langle\zeta_{0}^{2} \mid \zeta_{L}^{2}\right\rangle, a_{22}=\left\langle\zeta_{L}^{2} \mid \zeta_{L}^{2}\right\rangle$, where $\lambda_{0}, \lambda_{L}$ are the eigenvalues of the linearized GL equation [3], and $\left\langle f_{0} \mid f_{1}\right\rangle=\int f_{0} f_{1} d \vec{r} / V$. Besides the two trivial solutions $C_{L}=0, C_{0}=\lambda_{0} / a_{11}$ and $C_{0}=0$, $C_{L}=\lambda_{L} / a_{22}$ which correspond to the Meissner state and the giant vortex state, respectively, it is possible to have another solution with $C_{0}=\left(\lambda_{0} a_{22}-\lambda_{L} a_{12}\right) / D$, $C_{L}=\left(\lambda_{L} a_{11}-\lambda_{0} a_{12}\right) / D, \quad D=a_{11} a_{22}-a_{12}^{2} \quad$ corresponding to a multivortex state. The free energy of the ground state obtained using this approach is shown in Fig. 1 by the dashed curve, which is in good agreement with the results of our simulations (full curves). In order to discuss the multivortex $\leftrightarrow$ giant vortex transition, we analyzed the stability of the obtained solutions with respect to small perturbations of the coefficients $C_{0}, C_{L}$ (see Ref. [3]). The stability conditions for the multivortex state and the giant vortex state are $\lambda_{L}<\lambda_{\star}=\lambda_{0} a_{22} / a_{12}$ and $\lambda_{L}>\lambda_{\star}$, respectively. Consequently, for fixed $L$, there is a unique solution with a reversible transition from the multivortex state to the giant vortex, which occurs with an increasing magnetic field when $\lambda_{L}=\lambda_{\star}$. At this critical point the multivortex state coincides with the giant vortex state and consequently there is no jump in the magnetization. But the derivatives of the coefficients $C_{0}, C_{L}$ are discontinuous and correspondingly we expect a discontinuity in the first derivative of the magnetization. This is confirmed by our numerical simulations, where, in Fig. 3(b), the region around the $L=3$ multivortex to giant vortex transition is shown.

The magnetization of the disk $M=\int(H-$ $\left.H_{0}\right) d \vec{r} / 4 \pi V H_{c 2}$ with $R=4.8 \xi$ is shown in Fig. 4 for increasing (a) and decreasing (b) magnetic fields. Jumps in the magnetization correspond to transitions between states with a different number of vortices at the magnetic fields where the states cease to exist (see Fig. 1). With increasing disk thickness the demagnetization effect increases and as a result the transition points shift to larger magnetic fields, but the number of jumps remains the same. The average magnetic field in the disk can be estimated as $\langle H\rangle \approx H-4 \pi M$ and the relative magnetization $M / d$ as a function of $\langle H\rangle$ is "almost" a universal curve for all disk thicknesses [Fig. 4(c)]. For increasing magnetic field and disk thicknesses $d=0.2,0.4 \xi$ all transitions occur between giant vortex states. For the thinner disk $d=0.1 \xi$ we observe the following sequence of transitions $0 \rightarrow 1 \rightarrow 2_{g} \rightarrow 3_{m} \rightarrow 3_{g} \rightarrow 4_{m} \rightarrow 4_{g} \rightarrow 5_{g} \rightarrow 6_{g} \ldots$, where the lower index $(g, m)$ corresponds to giant and multivortex states, respectively. For a decreasing magnetic field multivortex states appear with a larger number of vortices. The sequence of transitions between multivortex states starts from $L=7$ and $L=8$ for $d=0.2 \xi$ and $d=0.1 \xi$, respectively. For the thicker disk $(d=0.4 \xi)$, a multivortex state appears only for $L=3,2$ just before the expulsion of a vortex from the disk. As is evident from Fig. 4(b), the appearance of

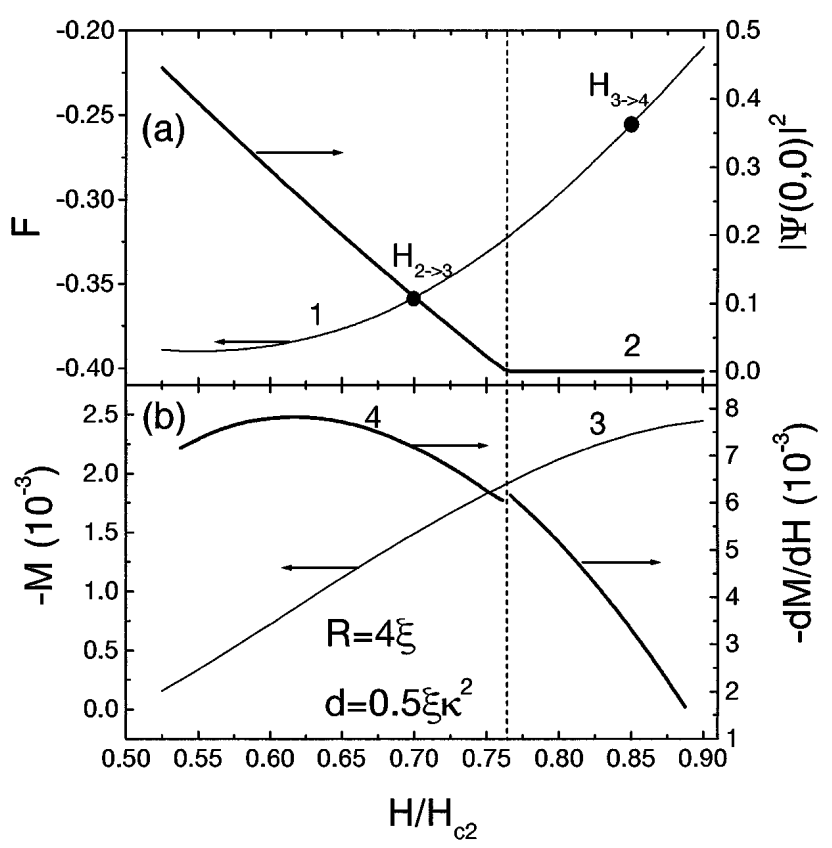

FIG. 3. The free energy (1), the square of the order parameter in the disk center (2), the magnetization (3), and its first derivative (4) for a disk with $R=4 \xi, d=0.5 \xi \kappa^{2}$ which is in the vortex state with $L=3$. The dashed vertical line shows the transition from the multivortex state to the giant vortex state. In (a) the solid circles indicate the points at which the equilibrium phase transitions $2 \rightarrow 3$ and $3 \rightarrow 4$ occur. 


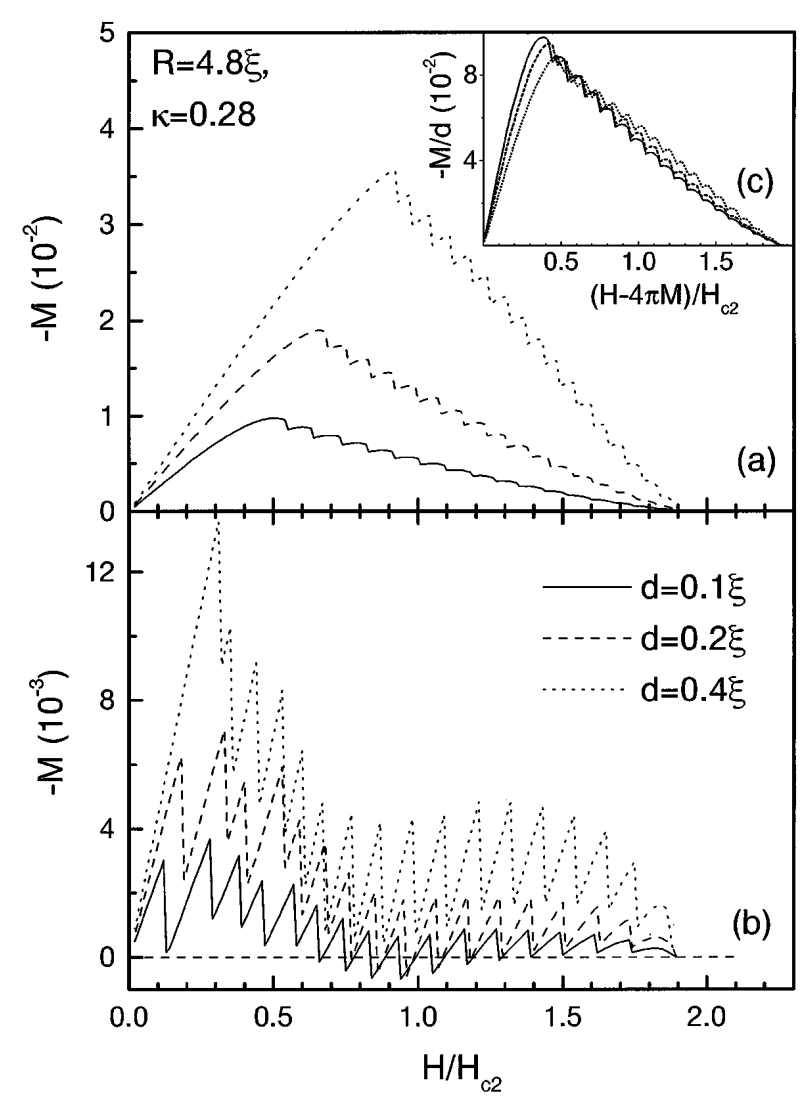

FIG. 4. Magnetization of a disk in increasing (a),(c) and decreasing (b) magnetic fields for $R=4.8 \xi, \kappa=0.28$, and different disk radii.

positive magnetization observed experimentally can also be explained within the 3D GL approach without the consideration of pinning effects. But the lowest energy state, i.e., the equilibrium state, has always a negative magnetization.

To distinguish quantitatively the giant vortex state from the multivortex states for the same number of vortices we consider the value of the order parameter $|\Psi|^{2}$ in the center of the disk. We find that this parameter, which is zero for a giant vortex state, goes almost linearly to zero when the magnetic field approaches some critical value [see Fig. 3(a), the thick curve]. Therefore, the magnetic field obtained by linearly interpolating $|\Psi(0,0)|^{2}$ to zero defines the transition from a multivortex state to a giant vortex state. Having the free energies of different vortex configurations we construct an equilibrium vortex phase diagram which is shown in Fig. 5 for two disk radii $R=4 \xi$ and $R=4.8 \xi$, respectively. The solid curves separate the regions with a different number of vortices and the dashed curves show the boundaries between the multivortex and the giant vortex states. For $L=1$ the single vortex state and the giant vortex state are identical. The shaded regions correspond to the multivortex states. The superconducting to normal transition occurs for $H / H_{c 2} \approx 1.9$ which is outside the plotted region. Notice that the multivortex area in the phase diagram reduces in

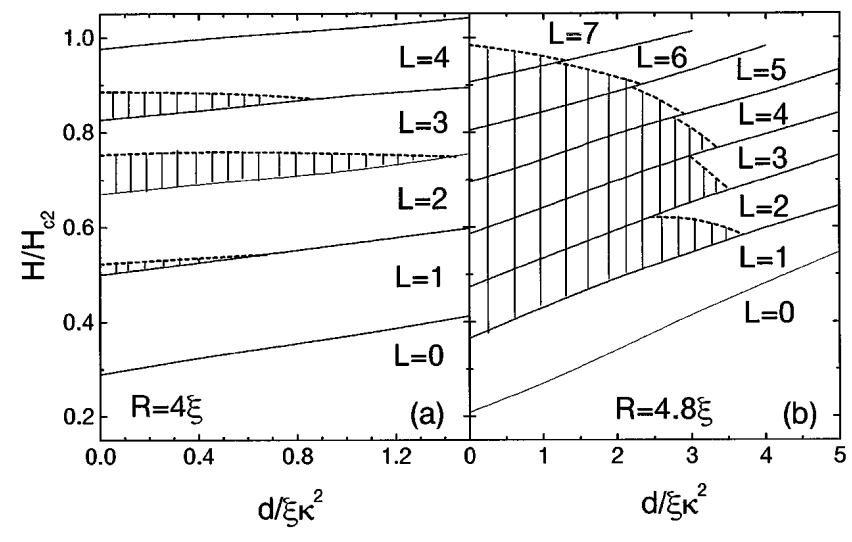

FIG. 5. The vortex phase diagram for two different disk radii $R=4 \xi$ (a) and $R=4.8 \xi$ (b). The shaded area corresponds to the multivortex state.

size with increasing disk thickness and it disappears in the limit of thick disks where only the giant vortex state survives. This behavior can be understood as follows: with increasing radius the energy difference between different $L$ states decreases and consequently it becomes possible to build a lower energy multivortex state out of a linear combination of giant vortex states. For a decreasing radius this is more difficult to do and there exists a critical radius below which no multivortex states have the lowest energy. To observe the multivortex $\leftrightarrow$ giant vortex transition one should investigate the derivative of the magnetization or the vortex configuration itself. Magnetization of individual disks has been measured by ballistic Hall magnetometers [1] which work as flux meters, while vortex configurations can be investigated using, e.g., a magnetic force microscope.

This work is supported by the Flemish Science Foundation (FWO-Vl) through Project No. G.0277.97, Project No. INTAS-93-1495-ext, and IUAP-IV.

*Permanent address: Institute of Theoretical and Applied Mechanics, Russian Academy of Sciences, Novosibirsk 630090, Russia.

†Electronic address: peeters@uia.ua.ac.be

[1] A. K. Geim et al., Nature (London) 390, 259 (1997).

[2] P.S. Deo et al., Phys. Rev. Lett. 79, 4653 (1997).

[3] V.A. Schweigert et al., Phys. Rev. B 57, 13817 (1998).

[4] P. G. de Gennes, Superconductivity of Metals and Alloys (Addison-Wesley, New York, 1989).

[5] V. M. Bedanov et al., Phys. Rev. B 49, 2667 (1994).

[6] A. L. Fetter, Phys. Rev. B 22, 1200 (1980).

[7] G. Bobel, Nuovo Cimento 38, 1741 (1966).

[8] A. I. Buzdin et al., Phys. Lett. A 196, 267 (1994).

[9] A. Bezryadin et al., J. Low Temp. Phys. 102, 73 (1996); M. Baert et al., Europhys. Lett. 29, 157 (1995).

[10] R. Kato et al., Phys. Rev. B 44, 6916 (1991).

[11] C. P. Bean et al., Phys. Rev. Lett. 12, 14 (1964).

[12] X. Zhang and J. C. Price, Phys. Rev. B 55, 3128 (1997).

[13] V. V. Moshchalkov et al., Phys. Rev. B 55, 11793 (1997). 